The Astrophysical Journal, 586:L127-L131, 2003 April 1

(C) 2003. The American Astronomical Society. All rights reserved. Printed in U.S.A.

\title{
THE FIRST MEASUREMENT OF SPECTRAL LINES IN A SHORT-PERIOD STAR BOUND TO THE GALAXY'S CENTRAL BLACK HOLE: A PARADOX OF YOUTH
}

\author{
A. M. Ghez,${ }^{1,2}$ G. Duchêne, ${ }^{1}$ K. Matthews,${ }^{3}$ S. D. Hornstein,,${ }^{1}$ A. Tanner,,${ }^{1}$ J. Larkin, ${ }^{1}$ M. Morris, ${ }^{1}$ E. E. Becklin, ${ }^{1}$ \\ S. Salim, ${ }^{1}$ T. Kremenek, ${ }^{1,4}$ D. Thompson, ${ }^{3}$ B. T. Soifer, ${ }^{3}$ G. Neugebauer, ${ }^{3,5}$ and I. McLean ${ }^{1}$ \\ Received 2002 December 21; accepted 2003 February 17; published 2003 March 12
}

\begin{abstract}
We have obtained the first detection of spectral absorption lines in one of the high-velocity stars in the vicinity of the Galaxy's central supermassive black hole. Both Br $\gamma(2.1661 \mu \mathrm{m})$ and $\mathrm{He} \mathrm{I}(2.1126 \mu \mathrm{m})$ are seen in absorption in S0-2 with equivalent widths $(2.8 \pm 0.3$ and $1.7 \pm 0.4 \AA)$ and an inferred stellar rotational velocity $\left(220 \pm 40 \mathrm{~km} \mathrm{~s}^{-1}\right)$ that are consistent with that of an O8-B0 dwarf, which suggests that it is a massive $\left(\sim 15 M_{\odot}\right)$ young (less than $\left.10 \mathrm{Myr}\right)$ main-sequence star. This presents a major challenge to star formation theories, given the strong tidal forces that prevail over all distances reached by S0-2 in its current orbit (130-1900 AU) and the difficulty in migrating this star inward during its lifetime from farther out where tidal forces should no longer preclude star formation. The radial velocity measurements $\left(\left\langle v_{z}\right\rangle=-510 \pm 40 \mathrm{~km} \mathrm{~s}^{-1}\right)$ and our reported proper motions for S0-2 strongly constrain its orbit, providing a direct measure of the black hole mass of 4.1 $( \pm 0.6) \times 10^{6}\left(R_{0} / 8 \mathrm{kpc}\right)^{3} M_{\odot}$. The Keplerian orbit parameters have uncertainties that are reduced by a factor of 2-3 compared to previously reported values and include, for the first time, an independent solution for the dynamical center; this location, while consistent with the nominal infrared position of Sgr A*, is localized to a factor of 5 more precisely ( \pm 2 mas). Furthermore, the ambiguity in the inclination of the orbit is resolved with the addition of the radial velocity measurement, indicating that the star is behind the black hole at the time of closest approach and counterrevolving against the Galaxy. With further radial velocity measurements in the next few years, the orbit of S0-2 will provide the most robust estimate of the distance to the Galactic center.
\end{abstract}

Subject headings: black hole physics - Galaxy: center - Galaxy: kinematics and dynamics -

infrared: stars — techniques: high angular resolution — techniques: spectroscopic

\section{INTRODUCTION}

Large ground-based telescopes present us with the opportunity to obtain an unprecedented view of the Galactic center in terms of both sensitivity and angular resolution. Initial studies of the Galaxy's central cluster at high angular resolution relied upon speckle imaging techniques. This first round of experiments was able to measure stellar motions on the plane of the sky, yielding estimates of the projected velocities (Eckart \& Genzel 1996; Ghez et al. 1998), projected accelerations (Ghez et al. 2000; Eckart et al. 2002), and three-dimensional orbital motions (Schödel et al. 2002; Ghez et al. 2003), which each provided a successively stronger case for a supermassive black hole at the center of the Milky Way and its association with the unusual radio source Sgr A* (Lo et al. 1985).

The advent of adaptive optics (AO) makes it practical to obtain spectroscopic observations of individual stars in the central $1^{\prime \prime} \times 1^{\prime \prime}$ of the Galaxy. Such measurements in principle provide the critical third dimension of motion, as well as astrophysical information about these high-velocity stars, such as spectral type and rotational velocity. While speckle spectroscopy was at-

\footnotetext{
${ }^{1}$ Department of Physics and Astronomy, University of California at Los Angeles, Box 951547, Los Angeles, CA 90095-1562; mclean@astro.ucla.edu, ghez@astro .ucla.edu, duchene@astro.ucla.edu, seth@astro.ucla.edu, tanner@astro.ucla.edu, larkin@astro.ucla.edu, morris@astro.ucla.edu, becklin@astro.ucla.edu, samir @astro.ucla.edu, kremenek@astro.ucla.edu.

${ }^{2}$ Institute of Geophysics and Planetary Physics, University of California at San Diego, 9500 Gilman Drive, La Jolla, CA 92093-0225.

${ }^{3}$ Caltech Optical Observatories, California Institute of Technology, MS 32047, Pasadena, CA 91125; gxn@irastro.caltech.edu, kym@irastro.caltech.edu, djt@irastro.caltech.edu, bts@irastro.caltech.edu.

${ }^{4}$ Department of Computer Science, Stanford University, 353 Serra Mall, Stanford, CA 94305-9025.

${ }^{5}$ Steward Observatory, University of Arizona, 933 North Cherry Avenue, Tucson, AZ 85721-0065.
}

tempted by Genzel et al. (1997), it was limited in spectral resolution to $R \sim 35$ and by source confusion. The first AO-assisted spectroscopy of the Galactic center was attempted with significantly higher spectral resolution, $R \sim 2000$ (Gezari et al 2002). Nonetheless, neither of these experiments yielded any line detections in the high-velocity (greater than $1000 \mathrm{~km} \mathrm{~s}^{-1}$ ) stars, indicating that the spectral lines are weak and would require either even higher spectral resolution or higher signal-to-noise ratio $(\mathrm{S} / \mathrm{N}$; or possibly both) to be detected.

In this Letter, we report the first measurement of spectral absorption lines in one of the high-velocity stars, S0-2. Section 2 describes the observations, $\S 3$ details the results, and $\S 4$ discusses how these observations improve the orbital solutions and raises questions regarding the origin of this apparently massive young star.

\section{OBSERVATIONS AND DATA ANALYSIS}

On the nights of 2002 June 2-3 (UT), near-infrared spectra of S0-2 were obtained with the W. M. Keck $10 \mathrm{~m}$ telescope's AO system (Wizinowich et al. 2000) and the Near-Infrared Camera 2 (NIRC2), the facility near-infrared instrument (K. Matthews et al. 2003, in preparation). AO corrections were made with a $R=13.2$ mag natural guide star (USNO 0600-28579500) located $30^{\prime \prime}$ away from S0-2. Using NIRC2 with its mediumresolution grism, 2 pixel slit, and medium-resolution pixel scale of $\sim 00^{\prime \prime} 02 \mathrm{pixel}^{-1}$, we achieved a spectral resolution of $R \sim$ $4000\left(\sim 75 \mathrm{~km} \mathrm{~s}^{-1}\right)$ and a spectral range of $0.3 \mu \mathrm{m}$ across the $1024 \times 1024$ array. On the first night, the $K$ bandpass filter was used with the slit positioned such that the spectral coverage was 2.04-2.30 $\mu \mathrm{m}$. Seven 20 minute exposures were obtained with S0-2 at various locations on the slit. HD 195500 (spectral type A1 V) and HD 193193 (spectral type G2 V) were observed as calibration sources at the same locations on the slit as S0-2. On 


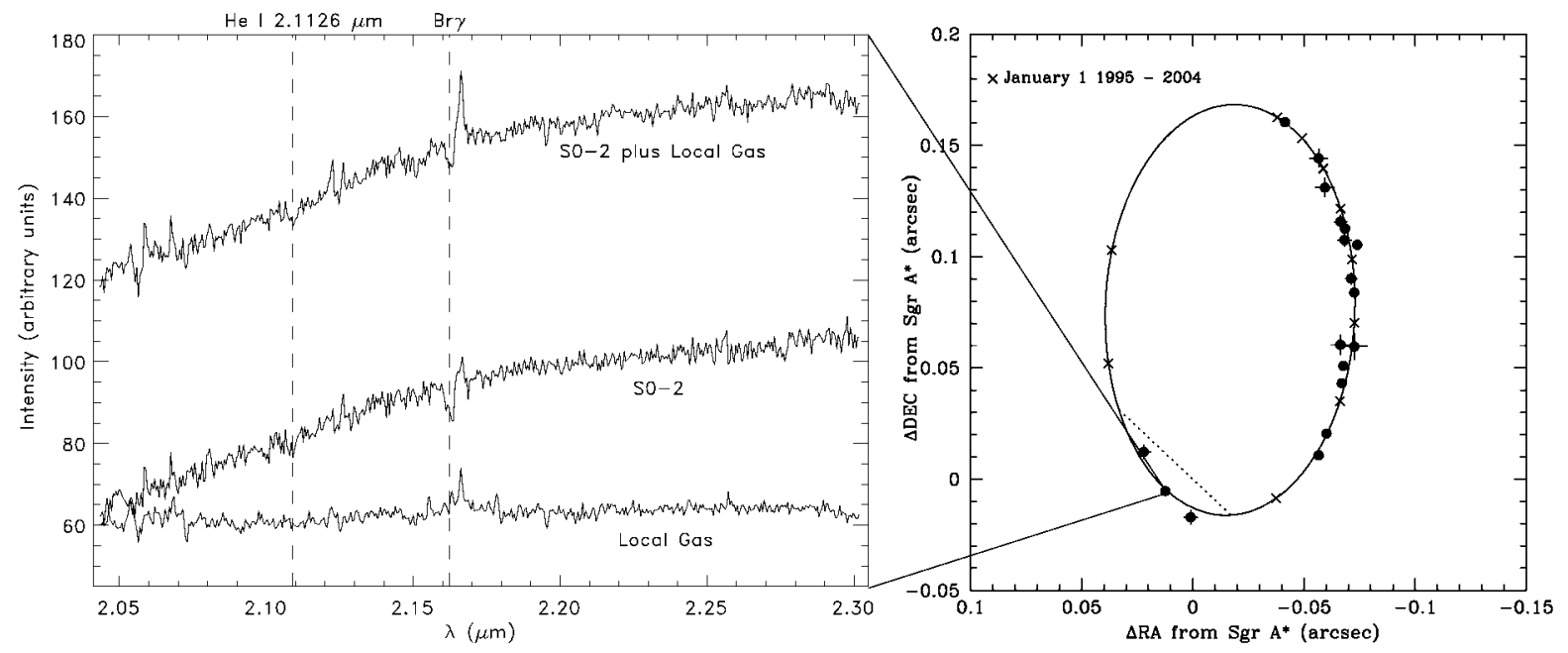

FIG. 1.-Left: First spectrum of S0-2 to show detectable photospheric absorption lines (Br $\gamma$ and $\mathrm{He}$ I $[2.1126 \mu \mathrm{m}])$. The final spectrum (middle) is the raw spectrum (top; with only an instrumental background removed) minus a local sky (bottom). The horizontal dimension has been rebinned by a factor of 2 for display purposes only. The vertical lines are drawn at 2.10899 and $2.16240 \mu \mathrm{m}$, which correspond to the locations of $\mathrm{Br} \gamma$ and $\mathrm{He}$ I for a $V_{\mathrm{LSR}}$ of $-513 \mathrm{~km} \mathrm{~s}{ }^{-1}$. This spectrum was obtained in 2000 June at the same time as one of the proper-motion measurements reported by Ghez et al. (2003) and shown in the right panel (filled circles). The crosses mark January 1 of each year between 1995 and 2004 for the best-fit orbit solution (solid line), which is based on both the radial velocity and the proper motions. The dotted line is the line of nodes, which reveals S0-2 to be behind the black hole for a mere $\sim 0.5 \mathrm{yr}$ out of its $15 \mathrm{yr}$ orbit.

the second night, the setup was altered somewhat to help identify and minimize systematic effects, such as fringing, which might complicate any line detections. The filter was changed to the $K^{\prime}$ bandpass, the slit was moved, giving a spectral coverage of 2.03-2.29 $\mu \mathrm{m}$, and the calibration sources observed were HD 190285 (spectral type A0 V) and HD 193193. Four spectra of S0-2 were obtained with this setup. Because of the high stellar densities at S0-2's location, a dark patch of sky $1 \mathrm{hr}$ east of Sgr $\mathrm{A}^{*}$ was observed on both nights to measure a purely sky background. The wavelength solution was obtained by identifying a set of $16 \mathrm{OH}$ emission lines in the spectra of the sky and by fitting a low-order polynomial function to the location of those lines throughout the detector along the spatial direction. The accuracy of this process is $\sim 9 \mathrm{~km} \mathrm{~s}^{-1}$, as measured by the dispersion of the residual of the fit.

The sky-subtracted spectrum of S0-2 in each data set was extracted over a 0 ."06 spatial interval along the slit and divided by the spectrum of the A-type star, extracted over the same window, to properly correct for the instrumental spectral response. Prior to this step, the spectrum of the G-type star was used to remove the strong $\mathrm{Br} \gamma$ absorption feature in the spectrum of the A-type star (Hanson, Conti, \& Rieke 1996). The spectrum was then divided by a blackbody of the temperature

TABLE 1

Spectral Properties of S0-2 ${ }^{\mathrm{a}}$

\begin{tabular}{|c|c|c|c|}
\hline Spectral Line & $\begin{array}{l}\text { EW } \\
(\AA)\end{array}$ & $\begin{array}{c}V_{z} \\
\left(\mathrm{~km} \mathrm{~s}^{-1}\right)\end{array}$ & $\begin{array}{c}V_{\text {rot }} \\
\left(\mathrm{km} \mathrm{s}^{-1}\right)\end{array}$ \\
\hline \multicolumn{4}{|l|}{$\operatorname{Br} \gamma(2.1661 \mu \mathrm{m}):$} \\
\hline Average spectrum & $2.8 \pm 0.3$ & $-510 \pm 39$ & $238 \pm 62$ \\
\hline Jun 2 spectrum ... & $\ldots$ & $-495 \pm 36$ & $\ldots$ \\
\hline Jun 3 spectrum .... & $\ldots$ & $-532 \pm 44$ & \\
\hline He I $(2.1125 \mu \mathrm{m}) \ldots$ & $1.7 \pm 0.4$ & $-532 \pm 96$ & $216 \pm 53$ \\
\hline He II $(2.1891 \mu \mathrm{m}) \ldots \ldots \ldots$ & $<0.5$ & $\ldots$ & $\ldots$ \\
\hline $\mathrm{N}$ III $(2.1155 \mu \mathrm{m}) \ldots \ldots \ldots$ & $<1.0$ & $\ldots$ & $\ldots$ \\
\hline He I $(2.0587 \mu \mathrm{m}) \ldots \ldots \ldots$ & $<1.3$ & & \\
\hline Average $\ldots \ldots \ldots \ldots \ldots \ldots$ & $\ldots$ & $-513 \pm 36$ & $224 \pm 40$ \\
\hline
\end{tabular}

${ }^{\mathrm{a}}$ For all lines except $\mathrm{Br} \gamma$, only the results from the averaged spectrum are listed. matching the spectral type of the calibrator to provide a spectrum corrected for all telluric absorption features.

The extracted spectra of S0-2 were still partially contaminated by background emission due to the gas present around the Galactic center as well as to the presence of the nearby bright source IRS 16C, whose wings extend along the spatial direction up to the location of S0-2. To correct for this emission, we estimated a local background by averaging the flux in two 0.06 spatial intervals along the slit located 0.75 to the southwest and 0.4 to the northeast of S0-2. This background estimate, which amounts to $40 \%-50 \%$ of the level of S0-2's continuum, was subtracted from the spectrum of the source to provide the final spectrum, which has a S/N pixel ${ }^{-1}$ of $\sim 30-80$ in the continuum (see Fig. 1). There is significant $\mathrm{Br} \gamma$ emission from the gas surrounding the Galactic center, and the intensity of this line varies spatially. Close to S0-2, however, the wavelength of this feature does not vary, which helps to distinguish it from any potential photospheric feature. In general, as long as the magnitude of S0-2's Doppler shift is greater than $\sim 300 \mathrm{~km} \mathrm{~s}^{-1}$, the line emission from the gas is unlikely to cause problems, either nondetections or biases, for $R \sim 4000$ measurements of stellar $\mathrm{Br} \gamma$ absorption. The zone of gas contamination is estimated on the basis of the structure of the $\mathrm{Br} \gamma$ emission line detected in the gas close to S0-2. The residual emission feature seen in the final spectrum of S0-2 corresponds to this local gas emission, and we believe that it is not physically associated with the star (see § 3 ).

\section{RESULTS}

The spectrum of S0-2, shown in Figure 1, has two identifiable spectral lines. These are both seen in absorption and are identified as the $\mathrm{H} \mathrm{I} \mathrm{(4-7)} \mathrm{or} \mathrm{Br} \gamma$ line at $2.166 \mu \mathrm{m}$ and the $\mathrm{He}$ I triplet at $2.1126 \mu \mathrm{m}\left(3 p^{3} P^{o}-4 s^{3} S\right)$, which is a blend of three transitions at $2.11274,2.11267$, and $2.11258 \mu \mathrm{m}$. Table 1 summarizes the properties of these two lines, which are obtained by fitting the background continuum over the whole spectrum with a loworder polynomial and fitting the lines with a Gaussian profile. 
Also given in Table 1 are limits on three lines that are not detected. The reported values and uncertainties are based on the fits to the average of all 11 spectra and the standard deviation of the mean of the fits to five independent pairs of spectra. The locations of the Gaussian peaks provide estimates of S0-2's Doppler shift, after a correction of $18 \mathrm{~km} \mathrm{~s}^{-1}$ is applied to account for the Earth's motion around the Sun and the Sun's motion toward the center of the Galaxy (Binney \& Merrifeild 1998). The agreement between the Doppler shifts inferred from the $\mathrm{Br} \gamma$ and He I absorption line profiles supports the interpretation of the Br $\gamma$ emission in S0-2's spectrum as residual gas emission as opposed to part of a P Cygni line profile. The weighted average of these two Doppler shift measurements yields a radial velocity of $-510 \pm 40 \mathrm{~km} \mathrm{~s}^{-1}$ for S0-2 during 2002.4187. Combining this radial velocity with the tangential velocity measured by Ghez et al. (2003) at the same epoch results in a total space motion of $6660 \pm 730 \mathrm{~km} \mathrm{~s}^{-1}$.

The Gaussian half-width at half-maximum (HWHM) values provide estimates of the rotational velocities. The intrinsic rotational profile of a star has a HWHM that corresponds to $90 \%$ of $v \sin i$ (Gray 1976). Since the HWHM of both lines is significantly larger than the instrumental HWHM $\left(\sim 38 \mathrm{~km} \mathrm{~s}^{-1}\right)$, we simply scale the Gaussian HWHM values by a factor of 1.1 to derive an estimated value of $v \sin i$. The values from the two lines are comparable and averaged together suggest a $v \sin i$ of $220 \pm 40 \mathrm{~km} \mathrm{~s}^{-1}$.

Equivalent widths (EWs) for the lines are also derived from the Gaussian fits. The EW (Br $\gamma)$ is $2.8 \pm 0.3 \AA$, and the EW (He I [2.1126 $\mu \mathrm{m}]$ ) is $1.7 \pm 0.4 \AA$. These values are checked against those obtained from integrating over $40 \AA$ windows centered on the lines, which provide similar results to within $3 \%-7 \%$. Using the same window and the Doppler shift measured for two detected lines, we derive the limits for He I $(2.0581 \mu \mathrm{m}), \mathrm{N}$ III $(2.1155 \mu \mathrm{m})$, and He II $(2.1885 \mu \mathrm{m})$, which are listed in Table 1.

Between our two consecutive nights of observations there is an expected change of $60 \mathrm{~km} \mathrm{~s}^{-1}$ in the radial velocity (§ 4.1). We therefore have also analyzed the spectra averaged over each night separately. The results for the $\mathrm{Br} \gamma$ line are reported in Table 1 . While the change in radial velocities between the two nights is not significant $\left[V_{z}(2002.4205)-\right.$ $V_{z}(2002.4177)=-37 \pm 47 \mathrm{~km} \mathrm{~s}^{-1}$ ], it is consistent with the predicted change. Furthermore, the EW and $v \sin i$ measurements from the spectrum averaged over the two nights appears to be unaffected by this shift. The individual nights' $V_{r}$ are used in the dynamical analysis in $\S 4.1$, and the averaged spectrum values of EW and $v \sin i$ are applied in the stellar astrophysics discussion in $\S 4.2$.

\section{DISCUSSION AND CONCLUSIONS}

\subsection{Dynamics}

The measurements of a radial velocity for $\mathrm{S} 0-2$ provide a new and powerful constraint on its orbit. S0-2's motion on the plane of the sky provided the first estimates of the orbital parameters (Schödel et al. 2002; Ghez et al. 2003). By combining the radial velocity with the proper motions reported by Ghez et al. and assuming a distance of $8.0 \mathrm{kpc}$ (Reid 1993), as was done in both proper-motion analyses, we obtain the first orbital solution for S0-2 based on measured three-dimensional motion (Table 2). We assume here that Sgr A* has no significant velocity with respect to the Galaxy along the line of sight, as is observed for its proper motion (Reid et al. 1999). Our
TABLE 2

Orbital SOLUTIONS FOR S0-2

\begin{tabular}{|c|c|c|}
\hline Orbital Parameter $^{\mathrm{a}}$ & This Work & Schödel et al. ${ }^{\mathrm{b}}$ \\
\hline$\Delta x_{0}$ (mas) & \pm 1.9 & $\ldots$ \\
\hline$\Delta y_{0}$ (mas) & \pm 1.4 & \\
\hline$M\left[\times 10^{6}\left(R_{0} / 8 \mathrm{kpc}\right)^{3} M_{\odot}\right] \ldots \ldots$ & $4.07 \pm 0.62$ & $3.7 \pm 1.5$ \\
\hline$A(\mathrm{mas}) \ldots \ldots \ldots \ldots \ldots \ldots \ldots$ & $125.6 \pm 5.5$ & $119 \pm 15$ \\
\hline$P(\mathrm{yr}) \ldots$ & $15.78 \pm 0.82$ & $15 \pm 1$ \\
\hline$e \ldots .$. & $0.8736 \pm 0$ & $0.87 \pm 0.03$ \\
\hline$T_{0}(\mathrm{y}$ & $2002.334 \pm 0.017$ & $2002.30 \pm 0.05$ \\
\hline$i(\operatorname{deg})$ & $-47.3 \pm 2.5$ & $\pm 46 \pm 4$ \\
\hline$\omega(\operatorname{deg})$. & $248.5 \pm 1.8$ & $250 \pm 5$ \\
\hline$\Omega(\operatorname{deg})$. & $49.9 \pm 3.0$ & $36 \pm 9$ \\
\hline
\end{tabular}

${ }^{\mathrm{a}}$ There are nine orbital parameters included in the fits reported here and seven in those calculated by Schödel et al. (2002), who fix the center of attraction (true focus) to the location of Sgr A* reported by Reid et al. (2003). $\Delta x_{0}$ and $\Delta y_{0}$ are the center of attraction's east-west and north-south offsets from Sgr $\mathrm{A}^{*}$; the reported uncertainty in the offsets includes only the uncertainty in the dynamical center. The remaining orbital parameters are semimajor axis $(A)$, period $(P)$, eccentricity $(e)$, time of periapse passage $\left(T_{0}\right)$, inclination $(i)$, angle of node to periapse $(\omega)$, and angle of line of nodes $(\Omega)$. While mass $(M)$ is not an independent parameter, it is reported here for convenience.

${ }^{\mathrm{b}} \mathrm{S} 0-2$ is referred to as S2 by Schödel et al.

fit produces a total mass estimate of $4.1( \pm 0.6) \times 10^{6}(D / 8 \mathrm{kpc})^{3}$ $M_{\odot}$, consistent at the $\sim 2 \sigma$ level with earlier estimates based on velocity dispersion measurements. Our results on S0-2's orbit do not assume the location of the dynamical center, as opposed to the analysis of Schödel et al. Despite these two additional free parameters, the uncertainties on the orbital parameters are reduced by a factor of $2-3$. This first orbital estimate of the Galaxy's dynamical center is not only consistent with the nominal infrared position of $\mathrm{Sgr} \mathrm{A}^{*}$ to within the uncertainties on the latter (Reid et al. 2003) but also a factor of 5 more precise ( \pm 2 mas).

The addition of radial velocity measurements also breaks the ambiguity in the inclination angle, $i$. With the proper-motion data alone, only the absolute value of the inclination angle can be determined, leaving the questions of the direction of revolution and where along the line of sight the star is located behind the black hole unresolved. Our radial velocity measurements indicate a negative inclination angle and consequently that S0-2 is counterrevolving both against the Galaxy and behind the black hole at the time of periapse. The improved location of the center of attraction from the orbital analysis results in a minimum offset of S0-2 from the black hole in the plane of the sky of $11 \pm 2$ mas, which is significantly larger than the expected Einstein radius $\left(\theta_{\mathrm{E}}=0.42\right.$ mas for the S02 distance behind the black hole of $\sim 100 \mathrm{AU})$ and therefore makes gravitational lensing an unlikely event (Wardle \& YusefZadeh 1992; Alexander \& Loeb 2001).

In principle, the addition of radial velocities to the study of S0-2's dynamics allows the distance to the Galactic center, $R_{0}$, to be a free parameter in the orbital fits (Salim \& Gould 1999). The measurements, however, were obtained just 30 days after the star's closest approach to the black hole, when the radial velocity was changing very rapidly (see Fig. 2). While the current radial velocity and proper-motion data set constrains $M / R_{0}^{3}$ very effectively ( $\sim 15 \%$ uncertainty), it does not yet produce a meaningful measurement of $R_{0}$. Nonetheless, as Figure 2 shows, the radial velocities from the currently allowed orbits quickly diverge, producing a spread of a few hundred $\mathrm{km} \mathrm{s}^{-1}$ in $1 \mathrm{yr}$.

Within the next few years, the orbital fits based on both proper motions and additional radial velocity measurements should provide the most direct and precise estimate of the 


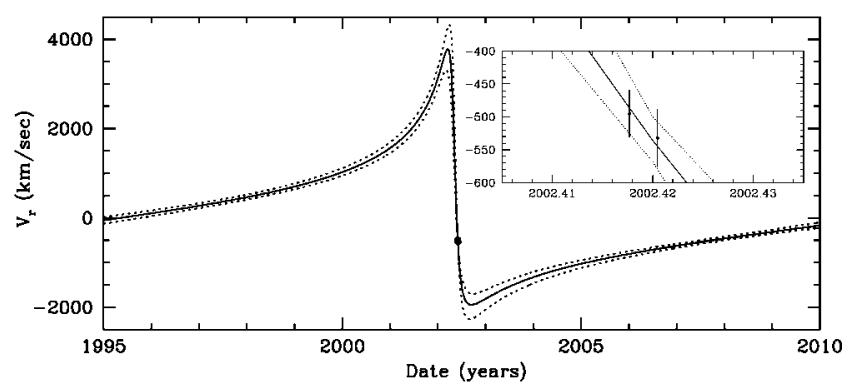

Fig. 2.-Measured radial velocity along with the predicted radial velocities. The solid curve comes from the best-fit orbit, and the dotted curves display the range for the orbital solutions allowed with the present data sets.

distance to the Galactic center, making it a fundamental rung in the cosmic distance ladder.

\subsection{Stellar Astrophysics}

The detection of absorption lines allows us to sort out the spectral classification ambiguities present when only photometric information is available and to determine if this star's photosphere has been altered as a result of its close proximity to the central black hole. The average brightness at $2.2 \mu \mathrm{m}$ for S0-2 is $K \sim 13.9 \mathrm{mag}$, and there is no evidence of brightening after periapse passage (Ghez et al. 2003). With a distance of $8.0 \mathrm{kpc}$ and $K$-band extinction of $3.3 \mathrm{mag}$ (Rieke, Rieke, \& Paul 1989), the $2.2 \mu \mathrm{m}$ brightness of S0-2 implies that if it is an ordinary star unaltered by its environment, it could be either an $\mathrm{O} 9$ mainsequence star or a K5 giant star; all supergiants are ruled out, as they are too bright by at least $2 \mathrm{mag}$ in the $K$ bandpass. Kleinmann \& Hall (1986) provide a 2.0-2.5 $\mu \mathrm{m}$ spectral atlas of late-type stars that demonstrates that if $\mathrm{S} 0-2$ is a $\mathrm{K} 5$ giant star, then it should have deep CO absorption lines, which definitively were not detected in either this experiment or our earlier experiment reported by Gezari et al. (2002). In contrast, the spectral atlas of $180 \mathrm{O}$ and B stars constructed by Hanson et al. (1996) shows that an O9 main-sequence star both lacks the CO absorption and has $\mathrm{Br} \gamma$ and $\mathrm{He} \mathrm{I}(2.1126 \mu \mathrm{m})$ consistent with the observed values. Futhermore, stars in this comparison sample earlier than $\mathrm{O} 8$ show $\mathrm{N}$ III $(2.115 \mu \mathrm{m})$ in emission and $\mathrm{He}$ II $(2.1885 \mu \mathrm{m})$ in absorption above our $3 \sigma$ thresholds, which are listed in Table 1; the lack of photospheric He I $(2.058 \mu \mathrm{m})$ absorption does not provide any additional constraints. Similarly, dwarf B-type stars later than B0 have absorption EWs that are too large. Together, the photometry and absorption-line EWs permit dwarf spectral types ranging from $\mathrm{O} 8$ to $\mathrm{B} 0$. Likewise, the rotational velocity of $224 \mathrm{~km} \mathrm{~s}^{-1}$ is reasonable for this range (Gatheier, Lamers, \& Snow 1981).

S0-2, therefore, appears to have a spectral type and hence temperature $(\sim 30,000 \mathrm{~K})$, as well as luminosity $\left(\sim 10^{3} L_{\odot}\right)$, that are consistent with a main-sequence star having a mass of $\sim 15 M_{\odot}$ and an age less than $10 \mathrm{Myr}$.

It is challenging to explain the presence of such a young star in close proximity to a supermassive black hole. Assuming that the black hole has not significantly affected S0-2's appearance or evolution, S0-2 must be younger than $10 \mathrm{Myr}$ and thus formed relatively recently. If it has not experienced significant orbital evolution, its apoapse distance of $1900 \mathrm{AU}$ implies that star formation is possible in spite of the tremendous tidal forces presented by the black hole, which is highly unlikely. If the star formed at larger distances from the black hole and migrated inward, then the migration would have to be through a very efficient process. Current understanding of the distribution of stars, however, does not permit such efficient migration. This problem is similar to that raised by the $\mathrm{He}$ I emission-line stars (e.g., Sanders 1992, 1998; Morris 1993; Morris, Ghez, \& Becklin 1999; Gerhard 2001; Kim \& Morris 2002), which are also counterrevolving against the Galaxy (Genzel et al. 1997), but amplifies it with a distance from the black hole that is an order of magnitude smaller. An alternative explanation for S0-2's hot photosphere is that it may be significantly altered by its environment.

While its periapse passage is too large for it to be tidally heated by the black hole as explored by Alexander \& Morris (2003), it may be affected by the high stellar densities found in this region. On the one hand, the high stellar densities might allow S0-2 to be an older giant star that has had its outer atmosphere stripped through collisions; however, to generate the necessary luminosity, significant external heating is required (Alexander 1999). On the other hand, high stellar densities might lead a cascade of merger events (Lee 1996), which would allow S0-2's formation process to have begun more than $10 \mathrm{Myr}$ ago. However, a large number of collisions would have had to occur to provide the necessary lifetime to bring it in from sufficiently large radii. More exotically, it could be a "reborn" star, which occurs as the product of a merger of a stellar remnant with a normal star. None of these possibilities is altogether satisfactory, leaving S0-2 as a paradox of apparent youth in the vicinity of a supermassive black hole.

We thank the staff of the Keck observatory, especially Randy Campbell, Grant Hill, Chuck Sorensen, David LeMignant, and director Fred Chaffee. This work has been supported by the National Science Foundation through the individual grant AST 99-88397 and the Science and Technology Center for Adaptive Optics, managed by the University of California at Santa Cruz under Cooperative Agreement AST 98-76783. The research of E. E. B., B. T. S., and D. T. is supported by NASA. The W. M. Keck Observatory is operated as a scientific partnership among the California Institute of Technology, the University of California, and the National Aeronautics and Space Administration. The Observatory was made possible by the generous financial support of the W. M. Keck Foundation.

\section{REFERENCES}

Alexander, T. 1999, ApJ, 527, 835

Alexander, T., \& Loeb, A. 2001, ApJ, 551, 223

Alexander, T., \& Morris, M. 2002, ApJL, submitted

Binney, J., \& Merrifield, M. 1998, Galactic Astronomy (Princeton: Princeton Univ. Press)

Eckart, A., \& Genzel, R. 1996, Nature, 383, 415

Eckart, A., Genzel, R., Ott, T., \& Schödel, R. 2002, MNRAS, 331, 917

Gathier, R., Lamers, H. J. G. L. M., \& Snow, T. 1981, ApJ, 247, 173

Genzel, R., Eckart, A., Ott, T., \& Eisenhauer, F. 1997, MNRAS, 291, 219

Gerhard, O. 2001, ApJ, 546, L39
Gezari, S., Ghez, A. M., Becklin, E. E., Larkin, J., McLean, I. S., \& Morris, M. 2002, ApJ, 576, 790

Ghez, A. M., Becklin, E. E., Duchene, G., Hornstein, S., Morris, M., Salim, S., \& Tanner, A. 2003, Astron. Nachr., in press

Ghez, A. M., Klein, B. C., Morris, M., \& Becklin, E. E. 1998, ApJ, 509, 678

Ghez, A. M., Morris, M., Becklin, E. E., Tanner, A., \& Kremenek, T. 2000, Nature, 407, 349

Gray, D. F. 1976, The Observation and Analysis of Stellar Photospheres (New York: Wiley-Interscience)

Hanson, M. M., Conti, P. S., \& Rieke, M. J. 1996, ApJS, 107, 281 
Kim, S. S., \& Morris, M. 2002, ApJ, submitted

Kleinmann, S. G., \& Hall, D. N. B. 1986, ApJS, 62, 501

Lee, H. M. 1996, IAU Circ. 169

Lo, K. Y., Backer, D. C., Ekers, R. D., Kellermann, K. I., Reid, M., \& Moran, J. M. 1985, Nature, 315,124

Morris, M. 1993, ApJ, 408, 496

Morris, M., Ghez, A. M., \& Becklin, E. E. 1999, Adv. Space Res., 23, 959

Reid, M. J. 1993, ARA\&A, 31, 345

Reid, M. J., Menten, K. M., Genzel, R., Ott, T., Schödel, R., \& Eckart, A. 2003, ApJ, in press (astro-ph/0212273)
Reid, M. J., Readhead, A. C. S., Vermeulen, R. C., \& Treuhaft, R. N. 1999, ApJ, 524, 816

Rieke, G. H., Rieke, M. J., \& Paul, A. E. 1989, ApJ, 336, 752

Salim, S., \& Gould, A. 1999, ApJ, 523, 633

Sanders, R. H. 1992, Nature, 359, 131 1998, MNRAS, 294, 35

Schödel, R., et al. 2002, Nature, 419, 694

Wardle, M., \& Yusef-Zadeh, F. 1992, ApJ, 387, L65

Wizinowich, P., Acton, S. D., Lai, O., Gathright, J., Lupton, W., \& Stomski, P. 2000, Proc. SPIE, 4007, 2 